\title{
Correction: Synergistic anti-AML effects of the LSD1 inhibitor T-3775440 and the NEDD8-activating enzyme inhibitor pevonedistat via transdifferentiation and DNA rereplication
}

Y. Ishikawa ${ }^{1}$, K. Nakayama ${ }^{1}$, M. Morimoto ${ }^{1}$, A. Mizutani ${ }^{1}$, A. Nakayama ${ }^{1}$, K. Toyoshima $^{1}$, A. Hayashi ${ }^{1}$, S. Takagi ${ }^{1}$, R. Dairiki ${ }^{1}$, H. Miyashita', S. Matsumoto ${ }^{2}$, K. Gamo', T. Nomura ${ }^{1}$ and K. Nakamura ${ }^{1}$

\section{Correction to: Oncogenesis}

https://doi.org/10.1038/oncsis.2017.76

Published online 11 September 2017

The FAB classification of OCI-M2, GF-D8, CMK-11-5, and THP-1 cell lines in Table 1 was wrong. This error has been fixed in the revised table.

Published online: 11 September 2019

Correspondence: K. Nakamura (kazuhide.nakamura@takeda.com)

'Oncology Drug Discovery Unit, Pharmaceutical Research Division, Takeda Pharmaceutical Company Limited, Fujisawa, Japan

${ }^{2}$ Integrated Technology Research Laboratories, Pharmaceutical Research Division, Takeda Pharmaceutical Company Limited, Fujisawa, Japan

These authors contributed equally: Y. Ishikawa, K. Nakayama 
Table 1 Synergy score of T-3775440/pevonedistat combination in an AML cell panel

\begin{tabular}{cccccc}
\hline Cell Line & Meaning & $\begin{array}{c}\text { Blending } \\
\text { Synergy }\end{array}$ & $\begin{array}{c}\text { Combination } \\
\text { Index }\end{array}$ & $\begin{array}{c}\text { Incubation } \\
\text { (h) }\end{array}$ & FAB \\
\hline TF-1a & Synergy & 36.5 & 0.3 & 72 & M6 \\
\hline TF-1a/Ara-C & Synergy & 34.9 & NA & 72 & M6 \\
\hline NB4 & Synergy & 33.5 & NA & 120 & M3 \\
\hline Kasumi-1 & Synergy & 31.8 & 0.45 & 120 & M2 \\
\hline MOLM16 & Synergy & 28.4 & NA & 120 & M7 \\
\hline HL-60/MX2 & Synergy & 23.3 & 0.42 & 120 & M2 \\
\hline HL-60 & Synergy & 19.6 & 0.41 & 120 & M2 \\
\hline HEL92.1.7 & Additivity & 14.6 & NA & 72 & M6 \\
\hline OCI-M2 & Additivity & 10.9 & 0.81 & 72 & M6 \\
\hline GF-D8 & Additivity & 8.6 & NA & 168 & M1 \\
\hline CMK11-5 & Additivity & 4.3 & 0.96 & 72 & M7 \\
\hline THP-1 & Additivity & -5.5 & NA & 144 & M5 \\
\hline OCI-AML3 & Additivity & -7.9 & NA & 168 & M4 \\
\hline EOL-1 & Additivity & -17.3 & NA & 120 & Eosinophilic \\
\hline CMK-86 & Subadditivity & -27.6 & 1.73 & 72 & M7 \\
\hline
\end{tabular}

NOTE: Combination index $(\mathrm{Cl})$ values in the range $0-0.7$ and $0.7-1.3$ are classified as synergy and additivity, respectively. When $\mathrm{Cl}$ values were not associated, nonlinear blending values greater than 20 and between -20 and +20 were classified as synergy and additivity, respectively. Heat maps are color-coded based on the combination effects: Green, synergy; orange, additive; red, subadditive. 